\title{
Gravity, Mass and the Cosmological Constant: All Can Have the Same Origin
}

\author{
Hubert J. Veringa ${ }^{1}$ \\ ${ }^{1}$ Emeritus Professor at Eindhoven University, The Netherlands \\ Correspondence: Hubert J. Veringa, Emeritus Professor at Eindhoven University, The Netherlands. E-mail: \\ veringa48@planet.nl
}

Received: March 1, 2018 Accepted: March 15, 2018 Online Published: April 16, 2018

doi:10.5539/jmr.v10n3p59 URL: https://doi.org/10.5539/jmr.v10n3p59

\section{Introduction}

The general belief about gravity is that any suitable theory should include, or will be, a merger of classical quantum theory and relativity. In a recent publication (Veringa, H. J., 2018) a new scheme of analysis for the mutual interaction between particles that have some exchange with respect to time and space has already been presented. The remarkable thing is that, apparently for more than one reason, particles will be interacting in groups of two and only two and can give rise to gravitational interaction. This pair formation was described quantum-mechanically.

This analysis starts from the from the classical Schrödinger equation to describe the behaviour of a pair of particles whose solution is used as the invariant term of the relativistic Einstein Energy equation, but this latter formulated in a quantum-mechanical setting known as the "Klein-Gordon" (KG) equation. By solving this equation the properties of the gravitational interaction, as they occur in Newton's gravity law, are found. Next to this it is also shown that the attribution of mass is a consequence of all other masses constituting our universe and whose calculated value is within the right order of magnitude of what is experimentally observed.

More or less as a surprise it became clear that the solution of the KG-equation, which already has lead to the law of gravity and particle masses, can be easily interpreted in such a way that the Cosmological Constant, as it has initially been introduced by Einstein in his famous Field Equation, emerges. This Constant appears to have basically the same properties as Einstein's Cosmological Constant. It became clear that the existence of such a constant is necessary to have a non-zero value of the gravitational interaction.

\section{Gravity, Mass and Cosmological Constant}

An important conclusion in an earlier document (Veringa, H. J., 2018) is that particles with a mass can be described as mutually non-interacting pairs containing only two members. This conclusion is expressed in the wave function:

$$
\psi_{i j}=\left(\frac{\alpha_{i j}}{r_{i j}}+\frac{\alpha_{j i}}{r_{j i}}\right) e^{i \beta_{i j} r_{i j}+i \beta_{j i} r_{j i}},
$$

in which the indices $(i j)$ represent the pair constituted by the masses $m_{i}$ and $m_{j} . \alpha_{k l}$ are amplitudes which will play an important role in the development of the theory. Important is the conclusion that $\alpha_{k l}=\alpha_{l k}$ meaning that the gravity interaction is symmetric. The origins for the coordinates $r_{k l}$ will have to be chosen carefully to quarantee sharp values for the pair momenta. $\beta_{k l}=-\beta_{l k}$ and have no influence on the $\alpha^{\prime} s$.

The wave function gives the presence of an entity to which a rest mass or energy, $m_{0 i j}$, can be attributed. In quantum-mechanical language, this rest mass becomes an operator and therefore it has to be multiplied by the wave function and its conjugated function: $\psi_{i j}^{*} m_{0 i j}^{2} \psi_{i j}$ and we get:

$$
\psi_{\mathrm{ij}}^{*} \mathrm{c}^{4} \mathrm{~m}_{0 \mathrm{ij}}^{2} \psi_{\mathrm{ij}}=\mathrm{c}^{4} \mathrm{~m}_{0 \mathrm{ij}}^{2}\left(\frac{\alpha_{\mathrm{ij}}}{\mathrm{r}_{\mathrm{ij}}}+\frac{\alpha_{\mathrm{ji}}}{\mathrm{r}_{\mathrm{ji}}}\right)^{2} .
$$

The individual members can make pairs with all other mass in their surroundings. This, already peculiar pair effect is used in the KG-equation which, in a quantum-mechanical representation, describes a field around these members:

$$
\varphi_{\mathrm{ij}} \varphi_{\mathrm{ji}}=\gamma_{\mathrm{ij}} \mathrm{r}_{\mathrm{ij}}^{-\mathrm{m}_{\mathrm{ijj}} \alpha_{\mathrm{ij}} / \mathrm{hc}} \gamma_{\mathrm{ji}} \mathrm{r}_{\mathrm{ji}}^{-\mathrm{m}_{0 \mathrm{ij}} \alpha_{\mathrm{ji}} / \hbar c}
$$


As before $\gamma_{k l}$ are the amplitudes for these wave functions.

The coordinates $r_{k l}$ have to be treated with care as it has been explained in several footnotes in the earlier publication (Veringa, H. J., 2018) .

The equation (2.3) contains all important information for the gravitational interaction, mass attribution and the Cosmological Constant.

\subsection{Gravity}

Inserting the solution (2.3) into the pair-based KG-equation leads to the following solution for the interaction energy $E_{i j}$ of the pair $(i j)$ :

$$
\left(E_{i j}^{2}+2 E_{i j} E_{j i}+E_{j i}^{2}\right) \varphi_{i j} \varphi_{j i}-c \hbar m_{0 i j}\left(\frac{\alpha_{i j}}{r_{i j}^{2}}+\frac{\alpha_{j i}}{r_{j i}^{2}}\right) \varphi_{j i} \varphi_{i j}=0
$$

which, by summing up over all pair-wise interactions of the elements out of which individual mass entities in a pair (12) are build up, leads to Newton's gravity law:

$$
\mathrm{E}_{12}=\mathrm{E}_{21}=\sqrt{2 \sigma \mathrm{ch}} \cdot\left(\mathrm{m}_{1} \mathrm{~m}_{2}\right) / \mathrm{R}
$$

The parameter $\sigma$ is a constant whose value is determined by the gravity constant $\mathrm{G}$ and equal to: $2.7 \times 10^{2} \mathrm{Jm} / \mathrm{kg}^{4}$, (Veringa, H. J., 2018) .

\subsection{Mass Attribution}

The field that occurs due to the KG-equation is not only present outside the particle but must also have its influence in areas where the particle mass density manifests itself. Not much is known about what this field inside the particle looks like and its local interactions, but the most simple approach would be to assume that the amplitude of the generator of this field (2.2) is constant. The dependence on space coordinates of this field inside the outer boundary of the particle then leads to the attribution of mass according to:

$$
\mathrm{m}_{\mathrm{p}}=\sum_{\mathrm{j}} \mathrm{m}_{0 \mathrm{ij}} \alpha_{\mathrm{ij}} / \mathrm{r}_{\mathrm{p}} \mathrm{c}^{2}=\left(\sigma / \mathrm{r}_{\mathrm{p}} \mathrm{c}^{2}\right) \sum_{\mathrm{j}} \mathrm{m}_{\mathrm{p}}^{2} \mathrm{~m}_{\mathrm{j}}^{2} \text {, or: } \mathrm{m}_{\mathrm{p}}=\mathrm{r}_{\mathrm{p}} \mathrm{c}^{2} / \sigma \sum_{\mathrm{j}} \mathrm{m}_{\mathrm{j}}^{2}
$$

This mass $m_{p}$ is found to be a consequence of all the interactions which the single particle has with the surrounding mass in which the distance, apparently, plays no role. Starting from $m_{p}=r_{p} c^{2} / \sigma \sum_{j} m_{j}^{2}$ and assuming that the mass of the universe is basically due to protons and neutrons with almost the same mass, so $m_{p}=m_{j}$, and assuming there are $N$ particles in the whole universe giving it a total mass of $M_{u}$ we can set:

$$
\mathrm{M}_{\mathrm{u}}=\mathrm{Nm}_{\mathrm{j}}=\mathrm{Nr}_{\mathrm{p}} \mathrm{c}^{2} / \sigma \sum_{\mathrm{j}} \mathrm{m}_{\mathrm{j}}^{2}=\mathrm{Nr}_{\mathrm{p}} \mathrm{c}^{2} / \sigma \mathrm{Nm}_{\mathrm{j}}^{2}=\mathrm{r}_{\mathrm{p}} \mathrm{c}^{2} / \sigma \mathrm{m}_{\mathrm{j}}^{2} .
$$

Estimates of the size of the universe on the basis of the inverse Hubble constant and the fact that the average intergalactic density is less than 100 baryons per cubic metre tell us that the total mass of the universe is of the order of $10^{54} \mathrm{~kg}$. $\sigma$ is calculated in paragraph 8 of (Veringa, H. J., 2018) at $2.7 \times 10^{2} \mathrm{Jm} / \mathrm{kg}^{4}$ and the proton mass is $1.7 \times 10^{-27} \mathrm{~kg}$ (Wikipedia.org/proton). It leads to an estimate for the $r_{p}$-value in the order of $10^{-16} \mathrm{~m}$, which is about a factor of 10 less than the experimentally derived size of a proton (0.8 femtometers) (Antognini, A., 2013). An electron which is 1840 times lighter than the proton will, according to equation (2.7), see the same surrounding as the proton, so its size would be smaller by the same factor.

Although the correspondence with measured data is of about the right order of magnitude, it is still a rough estimate and not without speculation. The total mass of the universe estimated on the basis of the Hubble constant, assuming a flat universe, should include gravitationally active dark matter. But if we would include the neglected term in the basic KG-equation and also take the dynamic mass into consideration we can easily explain the discrepancy.

\subsection{Cosmological Constant}

There is an interesting observation. If we play the same game with the solution (2.3) as we did before, we will get the following expression for the invariant term for a second KG-equation:

$$
\varphi_{\mathrm{ij}}^{*} \varphi_{\mathrm{ji}}^{*} \varphi_{\mathrm{ij}} \varphi_{\mathrm{ji}}=\gamma_{\mathrm{ij}}^{2} \gamma_{\mathrm{ji}}^{2} \mathrm{r}_{\mathrm{ij}}^{-2 \mathrm{~m}_{0 \mathrm{ij}} \alpha_{\mathrm{ij}} / \hbar \mathrm{hc}} \mathrm{r}_{\mathrm{ji}}^{-2 \mathrm{~m}_{0 \mathrm{ij}} \alpha_{\mathrm{ji}} / \mathrm{hc}}=\gamma_{\mathrm{ij}}^{2} \gamma_{\mathrm{ji}}^{2} \mathrm{r}_{\mathrm{ij}}^{-4 \mathrm{~m}_{0 \mathrm{ij}} \alpha_{\mathrm{ij}} / \hbar \mathrm{c}} \cong \gamma_{\mathrm{ij}}^{2} \gamma_{\mathrm{ji}}^{2} .
$$

In order to have gravity, this amplitude should be non-zero. Now consider a volume of space with a particle $m_{i}$ and the surroundings determined by its coordinate system as explained in footnote $*$ ). In this coordinate system the amplitudes $\gamma_{i j}^{2} \gamma_{j i}^{2}$ are occurring due to the particle $m_{i}$ and all other particles in the entire universe indicated by the index $j$. The 
influence due to the amplitude $\gamma_{i j}^{2} \gamma_{j i}^{2}$ does not change when the universe around becomes smaller or larger or whether the particles $m_{j}$ are moving around. Since $\varphi_{i j}^{*} \varphi_{j i}^{*} \varphi_{i j} \varphi_{j i}$ represents the invariant term in the second KG-equation, it is related to a mass density and therewith carries an energy density which we will call $\varepsilon_{\Lambda}$. We can solve the KG-equation with this invariant term or not, but in any case this energy will give rise to a pressure acting on $m_{i}$ which, different from a normal gas, does not change upon expansion or compression. Therefore and according to (Heacox, W. D.,2015) we can conclude that:

$$
\sum_{\mathrm{ij}} \gamma_{\mathrm{ij}}^{2} \gamma_{\mathrm{ji}}^{2} / 2=\varepsilon_{\Lambda}=\mathrm{c}^{4} \Lambda / 8 \pi \mathrm{G}=6.4 \times 10^{-10} \mathrm{~J} / \mathrm{m}^{3},
$$

in which $\Lambda$ is the Cosmological Constant as it is introduced by Einstein. As argued above the mass- or energy related entity due to the amplitude $\gamma_{i j}^{2} \gamma_{j i}^{2}$ behaves practically in the same way as the "Cosmological Constant" which Einstein introduced in his famous "Einstein Field Equation", but which he later called his "biggest blunder ever". The argument given here could, however, present a justification for this Cosmological Constant. Although this constant is under serious debate, so far it is still considered as the carrier of the dark energy and therewith the reason for the accelerated expansion of our universe (Heacox, W. D., 2015). In view of the argument given here one can say that the existence of a Cosmological Constant is necessary in order to have non-zero values for the amplitudes $\gamma_{i j}^{2} \gamma_{j i}^{2}$, and therefore the existence of gravity. It has to be remarked, however, that the equation (2.8) breaks down for very small values of $r_{i j}$ in such a way that the cosmological constant would increase asymptotically for $r_{i j}$ to zero.

*) The observer on particle $m_{i}$ sees the effect of the particle $m_{j}$ in its surroundings and coordinate system at the distance $r_{j i}$ and vice versa. This means that, formally, the momentum operators $\widehat{p_{l j}}, \widehat{p_{\jmath l}}$ work on the coordinates $r_{j i}, r_{i j}$ and therewith define the coordinate dependences on $r_{i j}$ and $r_{j i}$. So there are as many coordinate systems as there are particles, or mass carrying entities. The appreciation of this "kind of secret" code allows us to interpret gravity and mass attribution as two manifestations of one and the same mechanism. But in this latter respect equation (2.7) should be interpreted with caution. For instance, if we would squeeze $n$ particles in one the same coordinate system, we would see that the volume expands from $4 \pi r_{p}^{3} / 3$ to $4 \pi n^{3} r_{p}^{3} / 3$, whereas if we cluster these $n$ particles together the volume increases to $4 \pi n r_{p}^{3} / 3$.

\section{Conclusions}

1. By solving the Klein-Gordon equation for the pair represented as a single entity we arrive at the right form of Newton's law of gravity. Also by adding up the basic functions for single group of particles, or groups of particles, the right form of the gravity law between large bodies is obtained.

2. The Klein-Gordon equation is also found to be applicable at the level of a single particle and gives a value for its mass in dependence of all mass around in the entire universe. The calculated values are not far away from the experimentally derived numbers. The analysis given might open the gate for further research into the mystery of dark matter and dark energy.

3. The solution of the Klein-Gordon equation is such that an energy has to be dedicated to the pair-wise interaction leading to a repulsive force which, at a particle separation in a pair of more than atomic distances, has precisely the same properties as the Cosmological Constant in the Einstein Field equation.

4. Knowing the solution of the wave equation that describes the pair-wise interaction, gravity is needed to have a non-zero value for the amplitude of this wave equation. In the same way a Cosmological Constant is needed to attain non-zero values for the amplitude of the solution of the relativistic KG-equation.

\section{Acknowledgments}

The author wishes to express his gratitude to Klinkenberg BV, Zaanstad Netherlands https://www.klinkenbergbv.nl/ for their support to the work done.

\section{References}

Antognini, A. et. al. (2013).Science 339, 417-420.

Heacox, W. D. (2015). The Expanding Universe, Cambridge University Press.

Veringa, H. J. (2018). The Origin of Gravity, Journal of Mathematics Research, 10(1). http://www.ccsenet.org/journal/index.php/jmr/issue/view/1950.

Wikipedia.org/proton. 


\section{Copyrights}

Copyright for this article is retained by the author(s), with first publication rights granted to the journal.

This is an open-access article distributed under the terms and conditions of the Creative Commons Attribution license (http://creativecommons.org/licenses/by/4.0/). 